\title{
Bebidas embotelladas como fuentes adicionales de exposición a flúor
}

\author{
Juan Pablo Loyola-Rodríguez, C.D., Ph.D., ${ }^{(1)}$ Amaury de Jesús Pozos-Guillén, C.D., ${ }^{(2)}$ \\ Juan Carlos Hernández-Guerrero, C.D., Ph.D.(3)
}

\section{Loyola-Rodríguez JP, Pozos-Guillén AJ, Hernández-Guerrero JC. \\ Bebidas embotelladas como fuentes adicionales de exposición a flúor. Salud Publica Mex 1998;40:438-441.}

\begin{abstract}
Resumen
Objetivo. Evaluar y notificar la concentración de fluo ruro en bebidas embotelladas que se consumen en la ciudad de San Luis Potosí, S.L.P., México y su implicación como factor de riesgo a fluorosis dental. Material y métodos. Se estudió el contenido de algunos productos de las dos compañías refresqueras más importantes de México y de otras dos locales. Las muestras se recolectaron de 10 lotes diferentes en el caso de los refrescos y de cinco lotes en el de los jugos, con tres muestras por lote, durante tres meses. La determinación de concentración de fluoruro se realizó mediante el método del electrodo de ion selectivo. Resultados La concentración de flúor de muestras de diferentes plantas de compañías nacionales presentaron diferencias estadísticas significativas entre grupos $(p<0.05)$. La comparación de resultados entre compañías nacionales y locales también mostró diferencia estadística significativa $(p<0.05)$. Los diferentes jugos presentaron concentraciones de flúor elevadas. Conclusiones La mayoría de las bebidas embotelladas que se consumen en San Luis Potosí presentaron niveles de flúor por arriba de la norma mexicana $(0.7 \mathrm{ppm})$ y pueden ser un factor de riesgo adicional de fluorosis dental en su población.
\end{abstract}

Palabras clave: fluorosis dental; flúor; bebidas embotelladas; México

\author{
Loyola-Rodríguez JP, Pozos-Guillén AJ, \\ Hernández-Guerrero JC. \\ Bottled drinks as additional source \\ of fluoride exposition. \\ Salud Publica Mex 1998;40:438-441.
}

\begin{abstract}
A bstract
Objective.To evaluate and to report the concentration of flouride in soft drinks and juices consumed in San Luis Potosí, S.L.P., Mexico, and its implications as a risk factor for developing dental fluorosis. Material and methods The contents of some products from 2 main national companies and 2 other local companies were studied. The samples were collected from 10 different batches in the case of the soft drinks, and from 5 batches in the case of the juices, with 3 samples per batch, during 3 months. The ion selective electrode method was used to determine the concentration of fluoride. Results Soft drinks from 2 main national companies showed high fluoride concentrations that were statistically significant between groups $(p<0.05)$. Fluo ride levels of products from local and national companies also showed differences that were statistically significant between groups $(p<0.05)$. All natural juices tested showed high fluoride concentrations. Conclusions Most soft drinks and juices consumed in SLP had high fluoride levels above Mexican regulations ( $0.7 \mathrm{ppm}$ ) and could be a substantial risk factor for developing dental fluorosis.
\end{abstract}

Key words: dental fluorosis; fluoride; bottled drinks; Mexico a fluorosis dental es una hipoplasia del esmalte dentario inducida por la ingestión excesiva de fluoruro durante la etapa de formación de los dientes. Desde el punto de vista clínico, se pueden observar manchas que van desde un color blanquecino hasta un café oscuro, o incluso la pérdida de continuidad del esmalte. Los reportes de incidencia y prevalencia de fluorosis dental en México se han incrementado du-

(1) Director del Centro de Investigación en Estomatología, Facultad de Estomatología, Universidad Autónoma de San Luis Potosí (UASLP), México.

(2) Profesor asistente, Centro de Investigación en Estomatología, Facultad de Estomatología, UASLP, México.

(3) Coordinador del Laboratorio de Inmunología, División de Estudios de Posgrado e Investigación, Facultad de 0 dontología, Universidad N acional Autónoma de México, México.

Fecha de recibido: 6 de mayo de 1998 • Fecha de aprobado: 25 de junio de 1998

Solicitud de sobretiros: Dr. Juan Pablo Loyola Rodríguez. Centro de Investigación en Estomatología, Facultad de Estomatología, Universidad Autónoma de San Luis Potosí. Av. Dr. Manuel N ava 2, Zona Universitaria, 78290 San Luis Potosí, S. L. P., México. Correo electrónico: jloyola@ deimos.tc.uaslp.mx 
rante los últimos años, especialmente en las zonas centro y norte..$^{1-8}$ Tal es el caso de la ciudad de San Luis Potosí, S.L.P., localizada en un área donde el agua de consumo contiene cantidades excesivas de fluoruro en forma natural. ${ }^{9}$ En un estudio de 52 pozos municipales se detectaron niveles de fluoruro de entre $3.0 \mathrm{y}$ 4.0 ppm en $44 \%$ de ellos; de entre 1.0 y 2.0 ppm en $17 \%$; de entre 0.7 y 1.0 ppm en $16 \%$, y de menos de 0.7 ppm en $23 \%$ del total de pozos estudiados.

Sin embargo, la alta prevalencia de fluorosis dental en la ciudad de San Luis Potosí no puede atribuirse exclusivamente a los niveles de fluoruro en el agua de consumo; es necesario considerar otros factores de riesgo. $1,7,8$

Hervir el agua de consumo constituye un riesgo debido a que se incrementa en cerca de $66 \%$ la concentración inicial de flúor por la pérdida de volumen durante el proceso de ebullición. Otro problema es la falta de control de calidad que se tiene con la adición de fluoruro a la sal doméstica; en 1991 se dio inicio a un programa nacional de fluoración de sal doméstica con el objetivo de disminuir la incidencia de caries dental. Este tipo de sal no iba a ser distribuida en poblaciones donde el agua de consumo tuviera concentraciones mayores a $0.7 \mathrm{ppm}$ de flúor. Sin embargo, existen informes procedentes de algunos estados de la República, como es el caso de los estados de Chihuahua y San Luis Potosí, que indican lo contrario.,10 Por otra parte, las aguas de garrafón, los refrescos y los jugos de frutas pudieran considerarse como fuentes adicionales de exposición a fluoruro. En términos generales, el agua que se usa en la elaboración de refrescos proviene ya sea de la red municipal, o bien, de pozos profundos que se localizan en la propia fábrica, y en muchas ocasiones no existe un control adecuado de sales. El objetivo del presente trabajo fue evaluar y notificar la concentración de fluoruro en bebidas embotelladas (refrescos y jugos de frutas) que se consumen en la ciudad de San Luis Potosí y su implicación como factor de riesgo a fluorosis dental.

\section{Material y métodos}

Durante 1997 se estudió el contenido de algunos productos de las dos compañías refresqueras más importantes de México, procedentes de las plantas localizadas en los estados de San Luis Potosí, Jalisco y Zacatecas para la compañía 1, y en los estados de San Luis Potosí, Jalisco y Quéretaro para la compañía 2; además, se analizaron productos de dos compañías refresqueras locales, diferentes a las anteriores, ubicadas en la ciudad de San Luis Potosí, y una de jugos de frutas con planta en el estado de Querétaro. Las muestras se recolectaron de 10 lotes diferentes en el caso de los refrescos y de cinco lotes en el de los jugos, con tres muestras por lote, durante tres meses. La determinación de concentración de fluoruro se realizó mediante el método del electrodo de ion selectivo de fluoruro (electrodo combinado para flúor, 9609BN, Orion Research Incorporated, Boston, MA, USA). ${ }^{1}$ Con el propósito de lograr una mejor precisión en cada prueba, se preparó una curva de calibración de 0.1-5.0 ppm de fluoruro, yel control de aditividad se realizó con el estándar de referencia (Orion 040908); este procedimiento se llevó a cabo en cada experimento. En la lectura de todos los experimentos se empleó el potenciómetro modelo $720 \mathrm{~A}$ (Orion). Los resultados que se presentan en este trabajo se expresan como ppm de flúor y están clasificados de acuerdo con la compañía embotelladora. Se utilizaron las pruebas estadísticas ANOVA y $t$ de Student.

\section{Resultados}

En el cuadro I se pueden observar los resultados del análisis de las muestras de las compañías refresqueras más populares en el país. A todas luces la compañía 1 presenta grandes diferencias en concentraciones de flúor en sus distintas plantas; todas las concentraciones estuvieron por arriba del óptimo establecido por la norma mexicana para agua de consumo humano $(0.7$ $\mathrm{ppm}$ ). Las diferencias entre los grupos fueron estadísticamente significativas (ANOVA, $p<0.05$ ). Los resultados de la compañía 2 mostraron que los productos de la planta localizada en San Luis Potosí están dentro de los niveles óptimos de flúor; sin embargo, las muestras de las otras plantas indicaron concentraciones por arriba de lo permitido. Las diferencias entre los gru-

\section{Cuadro I \\ Niveles de fluoruro en refrescos de las PRINCIPALES COMPañías en el País. MÉxico, 1997}

\begin{tabular}{lllllll} 
& & \multicolumn{2}{c}{ Compañía 1 } & & \multicolumn{2}{c}{ Compañía 2 } \\
Estado & $n$ & $\bar{X} \pm \mathrm{DE}$ & Rango & & $\bar{X} \pm \mathrm{DE}$ & Rango \\
Jalisco & 10 & $1.38 \pm 0.55$ & $0.44-1.89$ & & $1.84 \pm 0.03$ & $1.82-1.88$ \\
\hline Zacatecas & 10 & $3.52 \pm 0.52$ & $3.18-4.12$ & & \\
\hline Q uerétaro & 10 & & & $1.41 \pm 0.18$ & $1.28-1.73$ \\
\hline San Luis Potosín & 10 & $3.04 \pm 0.14$ & $2.85-3.21$ & & $0.40 \pm 0.07$ & $0.33-0.56$ \\
n: número de muestras & & & & \\
\hline $\begin{array}{l}\text { X } \pm D \text { E: promedio } \pm \text { desviación estándar } \\
\text { N orma mexicana de contenido de flúor: } 0.7 \text { ppm }\end{array}$ \\
N ota: los resultados se expresan en ppm (partes por millón) de flúor
\end{tabular}


pos de la compañía 2 fueron estadísticamente significativas (ANOVA, $p<0.05$ ). La comparación de los resultados de las dos compañías mostraron diferencias significativas ( $t$ de Student, $p<0.05$ ).

Los resultados de las dos compañías refresqueras locales fueron los siguientes: una mostró niveles de fluoruro de $3.56 \pm 0.10$, con un rango de entre $2.85 \mathrm{y}$ $3.21 \mathrm{ppm}$, mientras que la otra registró niveles de $0.36 \pm 0.01$, con un rango de entre 0.35 y $0.37 \mathrm{ppm}$, de tal modo que se encontraron diferencias estadísticamente significativas entre ambas compañías ( $t$ de Student, $p<0.05$ ).

Finalmente, en el cuadro II puede observarse que todas las muestras estudiadas de la compañía de jugos de frutas tuvieron concentraciones de flúor por arriba de lo considerado como óptimo. Los niveles de flúor tuvieron un rango de 1.32-4.40 independientemente de la fruta utilizada para la elaboración del jugo, lo cual indica, sin lugar a dudas, que el nivel de flúor no depende del sabor de la fruta y sugiere que el agua usada en la elaboración del jugo tiene altas concentraciones de flúor.

\section{Discusión}

La sobrexplotación de mantos acuíferos que abastecen a varias ciudades de México ha tenido como consecuencia la necesidad de obtener agua potable de pozos cada vez más profundos. Esa situación ha teni-

\section{Cuadro II \\ Niveles de fluoruro en jugos de frutas. Méxıco, 1997}

\begin{tabular}{|c|c|c|c|}
\hline Sabor & $n$ & $\bar{X} \pm D E$ & Rango \\
\hline $\mathrm{N}$ aranja-papaya & 5 & $1.51 \pm 0.26$ & $1.32-1.81$ \\
\hline Frutas tropicales & 5 & $1.48 \pm 0.07$ & $1.48-1.62$ \\
\hline Frutas mixtas & 5 & $1.74 \pm 0.15$ & $1.57-1.86$ \\
\hline Manzana & 5 & $1.81 \pm 0.11$ & $1.68-1.90$ \\
\hline Uva & 5 & $2.00 \pm 0.19$ & $1.81-2.20$ \\
\hline $\mathrm{N}$ aranja-zanahoria & 5 & $2.16 \pm 0.34$ & $1.85-2.53$ \\
\hline $\mathrm{N}$ aranja-manzana & 5 & $2.13 \pm 0.16$ & $1.96-2.28$ \\
\hline $\mathrm{N}$ aranja & 5 & $2.19 \pm 0.77$ & $1.46-3.00$ \\
\hline Piña-zanahoria & 5 & $2.50 \pm 1.09$ & $1.30-3.42$ \\
\hline Uva-manzana & 5 & $3.15 \pm 1.00$ & $2.10-4.11$ \\
\hline Pera & 5 & $3.43 \pm 0.86$ & $2.74-4.40$ \\
\hline \multicolumn{4}{|c|}{$\begin{array}{l}\text { n: número de muestras } \\
\bar{X} \pm D E: \text { promedio } \pm \text { desviación estándar } \\
\text { N orma mexicana de contenido de flúor: } 0.7 \text { ppm }\end{array}$} \\
\hline \multicolumn{4}{|c|}{ N ota: los resultados se expresan en ppm (partes por millón) de flúor } \\
\hline
\end{tabular}

do como resultado que la concentración de fluoruro aumente debido a la precipitación de este ion en las profundidades de las fuentes de suministro de agua que abastecen a la población. Adicionalmente, en San Luis Potosí 50\% de las compañías que producen agua embotellada no tienen control de calidad en niveles de iones de fluoruro, lo cual refleja la contaminación existente en las fuentes de suministro de agua. ${ }^{8}$

La población mexicana es gran consumidora de bebidas gaseosas, lo cual puede ser una fuente potencial de riesgo de fluorosis dental. Las dos principales compañías en el país tienen poco control de la concentración de iones de flúor en algunas de sus plantas localizadas en diferentes estados de la Respública, exceptuando una ubicada en San Luis Potosí, que cumple con la concentración óptima. Esto último se debe, probablemente, a que la población obtiene información del problema a través de los medios masivos de comunicación lo cual, de alguna manera, ha presionado a los productores a tener un control; inclusive algunas compañías utilizan el control de iones de flúor de sus productos como fuente de promoción. No obstante, es necesario que el sector salud establezca un monitoreo periódico del contenido de flúor en las bebidas embotelladas, lo cual desafortunadamente no ha sucedido, debido a limitaciones técnicas. En ese sentido sería importante establecer convenios, de tal manera que las universidades locales pudieran colaborar en el proceso de monitoreo de esos productos.

En conclusión, la severidad de fluorosis dental en la ciudad de San Luis Potosí no puede atribuirse exclusivamente a la exposición a fluoruro en el agua de consumo, sino que deben considerarse otras fuentes de exposición como son la costumbre de hervir el agua que se bebe, la preparación de los alimentos con agua contaminada y el consumo de bebidas embotelladas (refrescos y jugos de frutas). Los altos niveles de flúor en bebidas embotelladas, independientemente de la localidad donde fueron elaboradas, muestran una relación directa con la contaminación de los diferentes mantos acuíferos de la región de donde se obtiene el agua para su elaboración. Por otra parte, los productos con concentraciones elevadas de flúor pueden venderse entre la población de otros estados del país que no tienen este problema.

\section{Agradecimientos}

Se agradece al doctor Fernando Díaz-Barriga Martínez su colaboración en la revisión del manuscrito y sus sugerencias para mejorar el proyecto. Investigación apoyada por CONACYT (ref. de proyecto F566). 


\section{Referencias}

1. Grimaldo M, Borja-A burto V, RamírezA, Ponce M, Rosas M, Díaz-Barriga F. Endemic fluorosis in San Luis Potosi, Mexico. I. Identification of risk factors associated with human exposure to fluoride. Environ Res 1995; 68:25-30.

2. Pozos A, Loyola-Rodríguez JP, Rueda A, Vázquez S. Fluorosis en dentición temporal. Memorias del I Concurso Estudiantil $\mathrm{N}$ acional de Investigación (CEN I). San Luis Potosí: Editorial Universitaria, 1996;1:14.

3. Lozano V. Fluorosis dental en Ensenada, Baja California. Rev ADM 1992;6:340-344.

4. De la Cruz D, Juárez N, Castillo L, Pérez F. Concentración y distribución de flúor en esmalte de dientes deciduos, estudio in vitro. Rev ADM 1994;2:98-101.

5. Barrandey S, Cabello M, Magaña J, Rodríguez E. Sal fluorada, riesgo 0 beneficio para la población de la ciudad de Chihuahua. Rev ADM1994; 2:80-89.
6. O rtiz M, Vargas D, O valle W. Fluorosis dental de la población escolar de Salamanca, Guanajuato. Memorias del I C oncurso Estudiantil N acional de Investigación (CEN I). San Luis Potosí: Edito rial U niversitaria, 1996;1:15. 7. Grimaldo M, Turrubiates F, Milán J, Pozos A, Alfaro C, Díaz-Barriga F. Endemic fluorosis in San Luis Potosi, Mexico. III. Screening for fluoride exposure with a geographic information system. Fluoride 1997;30:35-40. 8. Loyola-Rodríguez JP, Pozos A, Rueda A, Vázquez S, De La Paz G. Factores de riesgo a fluorosis dental en San Luis Potosí, México. Rev ADM 1996;6:295-300.

9. La minería en México. México, D.F.: IN EGI, 1996.

10. Díaz-Barriga F, N avarro-Q uezada A, G rijalva M, G rimaldo M, LoyolaRodríguez JP, D eogracias M. Endemic fluorosis in Mexico. Fluoride 1997; 30:223-239. 